Discussion: Based on our extensive search of the published literature, this is the first reported case in the U.S. rehabilitation literature of a spinal cord injury secondary to small cell carcinoma.

Conclusions: Although spinal cord injury secondary to neoplasia is well known, epidural and paraspinal small cell carcinoma is an unreported cause of spinal cord injury. Tumor growth of this rapidity is also unreported. Although the type of tumor is rare and highly aggressive, rehabilitation played an important role in maintaining the patient's independence, function, and quality of life.

\section{Poster 427}

\section{Successful Long-term Community Discharge After Rehabilitation of Delayed Radiation Myelopathy: A Case Report.}

Viet H. Vu, DO (Wayne State University; Rehabilitation Institute of Michigan, Detroit, MI); Lourdes A. Alfonso, MD; Edward C. Nieshoff, MD.

Disclosures: V. H. Vu, None.

Patients or Programs: A 38-year-old man with T2N2M0 laryngeal cancer.

Program Description: The patient was treated with a standard regimen of chemotherapy and radiation therapy (RT). After 17 months, he developed left sided weakness, requiring a cane to ambulate. A diagnosis of radiation necrosis was made after laminectomy and cord biopsy. His functional status was setup for feeding, maximal assistance for transfers and total assistance for toileting. The patient was admitted to IPR.

Setting: Acute rehabilitation hospital.

Results: After 6 weeks of IPR, manual muscle testing did not change. However, his function improved to modified independent with basic ADLs and standby assist for transfers. The patient's mother was trained in intermittent catheterization. He received a hospital bed, wheelchair, shower chair, and hemi-walker and was discharged home as a C2 ASIA D tetraplegic. In 4 months, he became C4 complete tetraplegic and developed a stage IV sacral pressure ulcer. He was readmitted for 2 weeks of IPR for family education on pressure ulcers then discharged home.

Discussion: RM is a complication of radiation therapy when the treatment window includes the spinal cord. Early RM is self limiting. Our patient presented with delayed RM despite radiation within the accepted safety margin of $50 \mathrm{~Gy}$. Symptoms occur 6-24 months after RT causing demyelination and vascular changes. Prophylaxis with hyperbaric oxygen, anticoagulation or steroids, are being studied. It is unfortunately true that patients with disseminated malignancies and associated cervical motor-complete cord lesions are often denied oncologic rehabilitation. However, our patient benefited significantly from inpatient rehabilitation, such that he was successfully discharged home with his family for over 2 years. This observation contradicts the therapeutic nihilism commonly seen in this setting. It supports consideration of inpatient rehabilitation for patients with cervical myelopathy associated with complications of advanced malignancy (such as delayed RM) in the presence of good family support.

Conclusions: Admission to inpatient rehabilitation can improve function and quality of life in patients diagnosed with cervical myelopathy associated with advanced malignancy.

\section{Poster 428}

\section{Successful Pregnancy and Delivery in a $\mathrm{Cl}$ ASIA A Spinal Cord Injured Woman: The Role of Coordinated Care Between PM\&R and Obstetrics Services: A Case Report.}

Cindy Y. Lin, MD (Stanford University Hospital, Stanford, CA); Stephen McKenna, MD; Kazuko L. Shem, MD.

Disclosures: C. Y. Lin, None.

Patients or Programs: 20-year-old pregnant woman with a Cl ASIA A SCI.

Program Description: 20-year-old woman involved in a motor vehicle accident who sustained a Cl ASIA A SCI, right $\mathrm{BKA}$, and sacral and bilateral pubic rami fractures. Patient became pregnant through normal conception 2 years after the injury. The patient is ventilator dependent on room air with a tracheostomy, has nutritional support via PEG feeds, neurogenic bowel and bladder with suprapubic catheter. Patient was followed up closely throughout the pregnancy by coordinated care between the PM\&R and High Risk Pregnancy Clinics. Pregnancy was complicated by gestational diabetes mellitus type Al.

Setting: Tertiary care hospital.

Results: At 33 weeks' routine follow-up, the patient was found to be in preterm labor with contractions every 1-2 minutes. Patient was admitted to L\&D Service. Rehabilitation service critical care staff was seconded to the L\&D service. The patient was administered magnesium sulfate for tocolysis, however, this was discontinued when she developed mild hypoxia. The decision was made to proceed with labor augmentation with Pitocin when patient could not be weaned from oxygen supplementation. Patient received an epidural to decrease risk of autonomic dysreflexia. During her labor, patient had blood pressures to $140 / 100$ and received IV hydralazine. The patient had a forceps-assisted delivery of a male infant in vertex presentation with Apgars of 6 at 1 minute and 8 at 5 minutes, birth weight $1622 \mathrm{~g}$.

Discussion: This is the first case, to our knowledge, documenting successful normal spontaneous vaginal delivery to a woman with Cl ASIA A SCI. Common complications in pregnant women with SCI include changes in ventilator needs, autonomic dysreflexia, urinary tract infections, incontinence, pressure ulcers, thrombosis, spasticity, and preterm labor.

Conclusions: Coordinated care between rehabilitation 